\title{
Human lactoferrin efficiently targeted into caprine beta-lactoglobulin locus with transcription activator-like effector nucleases
}

\author{
Yu-Guo Yuan ${ }^{1,2, a}$, Shao-Zheng Song ${ }^{1, a}$, Meng-Ming Zhu' ${ }^{1}$, Zheng-Yi He ${ }^{1}$, Rui Lu' ${ }^{1}$, Ting Zhang ${ }^{1}$, Fei Mi ${ }^{1}$, \\ Jin-Yu Wang ${ }^{1, *}$, and Yong Cheng ${ }^{1,2, *}$
}

\footnotetext{
* Corresponding Authors: Jin-Yu Wan

E-mail: jywang@yzu.edu.cn

Yong Cheng

Tel: +86-514-87979348, Fax: +86-514-87972218,

E-mail: cheng1391@hotmail.com

1 Jiangsu Co-Innovation Center for Prevention and Control of Important Animal Infectious Diseases and

Zoonosis/College of animal science and technology, Yangzhou University, Yangzhou, Jiangsu 225009,

China

2 Jiangsu Key Laboratory of Integrated Traditional

Chinese and Western Medicine for Prevention and

Treatment of Senile Diseases, Yangzhou, 225001,

China
}

a These authors contributed equally to this work. Submitted Sept 19, 2016; Revised Nov 11, 2016 Accepted Dec 13, 2016
Objective: To create genetically modified goat as a biopharming source of recombinant human lacotoferrin (hLF) with transcription activator-like effector nucleases.

Methods: TALENs and targeting vector were transferred into cultured fibroblasts to insert hLF cDNA in the goat beta-lactoglobulin (BLG) locus with homology-directed repair. The gene targeted efficiency was checked using sequencing and TE7I assay. The bi-allelic gene targeted colonies were isolated and confirmed with polymerase chain reaction, and used as donor cells for somatic cell nuclear transfer (SCNT).

Results: The targeted efficiency for BLG gene was approximately 10\%. Among 12 Bi-allelic gene targeted colonies, five were used in first round SCNT and 4 recipients $(23 \%)$ were confirmed pregnant at $30 \mathrm{~d}$. In second round SCNT, 7 (53\%), 4 (31\%), and 3 (23\%) recipients were confirmed to be pregnant by ultrasound on $30 \mathrm{~d}, 60 \mathrm{~d}$, and $90 \mathrm{~d}$.

Conclusion: This finding signifies the combined use of TALENs and SCNT can generate biallelic knock-in fibroblasts that can be cloned in a fetus. Therefore, it might lay the foundation for transgenic hLF goat generation and possible use of their mammary gland as a bioreactor for large-scale production of recombinant hLF.

Keywords: Beta-lactoglobulin; Gene Targeted; Transcription Activator-Like Effector Nucleases; Human Lacotoferrin; Goat; Nuclear Transfer

\section{INTRODUCTION}

Generation of genetically modified animals is a powerful tool for analyze functions, disease association and production of commercially important organisms of gene [1]. However, there are several limitations such as poor efficiency of somatic cell nuclear transfer (SCNT) of somatic cells targeted by homologous recombination (HR) and unavailability of authentic embryonic stem cells limited its application in domestic animals as well as in large scale industrial production. To the date, SCNT combined with HR is the only practiced technique in target gene editing (novel transgenic breeding) for domestic animals, which is relatively low efficient [2]. Other reported approaches designed for improving production of genetically modified animals targeting specific genomic sequences are zinc-finger nuclease (ZFN) [3-5] and transcription activator-like effector nucleases (TALENs) [6,7], which are potential next-generation platforms for customized genomic editing and transgenic animals production, as well as generation of transgenic cells lines in vitro. Unlike ZFNs and CRISPR/Cas9, TALENs have fewer off-target effects and lower toxicity [8].

Traditionally, goat's milk is an alternative to cow's milk, a source of nutrients and delicacy for humans, is considered as a palatable baby drink for its composition and better digestibility compared to cow's milk. However, milk and milk-based products contain allergens which are important sources of food borne allergy, thus the wide use of milk in food industry is restricted [9]. Beta- 
lactoglobulin (BLG) is a major milk protein and a well-known allergen [10], though enzymatically hydrolyzed whey proteins with hypoallergenic formulas have been developed in dairy industry to reduce the allergenic potential of BLG but it still remains a costly process and increases the manufacture costs. Alternatively, genetic modifications in big animals might facilitate the establishment of a BLG free animal and low cost production of allergens free milk $[11,12]$. Moreover, mammary gland is considered as the best available bioreactor, since milk is a source of raw material which is unprocessed, safe, abundant, renewable, easy to obtain and well accepted by consumers. BLG is also an ideal locus in mammary gland bioreactors, which potentially drive foreign gene(s) to especially express in the milk [11]. The mono-allelic genes knocked-in of BLG or $\beta$-casein have already been reported by in goat and cow [4,9], however, the expression of foreign protein in goat and cow milk using mono-allelic system did not show ideal result, instead it got even lower efficiency than that using a shorter-promotor region [13-16]. Only few studies of production of bi-allelic gene knocked-in BLG or $\beta$-casein livestock have been reported. The present research was to establiahe a bi-allelic gene knock-in system using human lactoferrin (hLF) cDNA, which was a multifunctional glycoprotein and had important application for food production and medical manufacturing. We demonstrated that targeted cells could possibly be used as nuclear donors in SCNT and are capable of directing development to at least $90 \mathrm{~d}$ of pregnancy by second round SCNT. Our results suggested that TALEN could be a useful tool in the production of gene-targeted goat.

\section{MATERIALS AND METHODS}

\section{Animal care}

The experimental procedure was approved by the Institutional Animal Care and Use Committee at Yangzhou University (2015. 1.1064).

\section{Construction of TALENs plasmids for gene targeting}

TALENs expression plasmids were assembled using TALEN Assembly Kit (SIDANSAI Biotechnology CO., LTD, Cat. No: 1803015, Shanghai, China). Two pairs of TALENs were designed to target exon 1 and exon 3 in the caprine BLG (Ensembl ID: Z33881.1). The BLG TALENs recognition sequences were as follows: (TALEN1) left TALEN 5'-CCAGACACCCACCTGT-3' and (TALEN2) right TALEN 5' - ACAGG TGGGTGTCTGG -3', and (TALEN3) left TALEN 5'-GTGCTCGA AGAAGAT-3' and (TALEN4) right TALEN 5'-CTTGAACACCGCAGGG-3', respectively. TALEN pairs were transfected into caprine fetal fibroblast to count the numbers of survival cell colonies and determine the targeting efficiency.

\section{Targeting vector construct}

Locus-specific homology arms were $3.7 \mathrm{~kb}(-3,781$ to -17$)$ and
$1.8 \mathrm{~kb}(+6,254$ to $+8,037)$ from BLG locus for $5^{\prime}$ arm and $3^{\prime}$ arm, respectively; and the hLF cDNA was $2.32 \mathrm{~kb}$. pBLC-TK construct was prepared by insertion of XhoI-XhoI fragment (4.37 kb) from plasmid Porf-hsv1tk (Invivogen, San Diego, CA, USA) into pBLC14 at the Sal I site [17], which was located downstream BLG 3'flanking sequence. The vector was constructed with a combination of standard enzyme restriction/ligation and In-Fusion cloning method and the target vector was linearized with Not I and Sal I before electroporation.

\section{Preparation of cells and culture conditions}

Caprine fetal fibroblasts were harvested from 35-day-old fetuses (or 43-day-old gene targeted fetuses). In brief, head, viscera and bones of each fetus were removed and washed three times using Dulbecco's phosphate buffered saline (D-PBS, Sigma, St. Louis, MO, USA). After that, the washed tissue was finely chopped into fragments smaller than $1 \mathrm{~mm}$ in size, and subsequently placed in a 35-mm culture dishes (Falcon, BD, Qume Drive, San Jose, CA, USA) incubated for $30 \mathrm{~min}$ in a humidified incubator at $37^{\circ} \mathrm{C}$ with $5 \% \mathrm{CO}_{2}$. Upon confirmation of adherence of the tissue fragments to the culture dish, a high-glucose Dulbecco's modified Eagle medium (Sigma, USA) containing 10\% fetal calf serum (HyClone, Beijing, China) was added gently. At 2 to 3 days, the fragments of tissue were removed, and the adherent cells to the bottom of the culture dish were continued to culture. During one to three passages, the cells were frozen and stored according to conventional methods.

TALEN transfection and evaluation of targeting efficiency For TALENs transfection, $1.5 \times 10^{5}$ cells were seeded per well in a 6 -well plate. On next day, the cell concentration was adjusted to $5 \times 10^{6}$ cells $/ \mathrm{mL}$ and $15 \mu \mathrm{g}$ of each TALEN expression plasmid (in pairs) were added to the cells. Then, $200 \mu \mathrm{L}$ of the transfection mixture was added to a 2-mm gap electroporation cuvette and were electroporated with Eppendorf multioporator (400 V, 300 $\mu$ s, one pulse). To evaluate the mutation efficiency, DNA was extracted at $24 \mathrm{~h}$ post-transfection from the mixed cells $\left(10^{6}\right.$ cells) for polymerase chain reaction (PCR) and sequencing experiments. Primer locations are shown in Figure 1: T1 (TGGCTCT GACCTGCCCTGT), T2 (CCCTAGCTG ACTGATGCGAAC); T3 (5'-TCTGGCTTCATTTGACTTCTC-3'), T4 (5'-TTT CTC ATCTCGGATTTAGGAC-3'). Mutations were also assessed by the T7 endonuclease I (T7EI) assay [18,19]. Briefly, purified PCR products from selected colonies and wild-type cells (200 ng for each) were denatured at $95^{\circ} \mathrm{C}$ for $5 \mathrm{~min}$, re-annealed at room temperature for $10 \mathrm{~min}$, and digested by T7EI (Beijing View Solid Biotech, Beijing, China) at $37^{\circ} \mathrm{C}$ for $30 \mathrm{~min}$. Different bands were expected for mutant colonies, the TALENs plasmid transfection experiment was performed at least in triplicates. Analyze the reaction products by agarose gel electrophoresis and quantify the band intensities by using Image J. Nuclease activity can be quantified by the proportion of digested and undigested PCR 


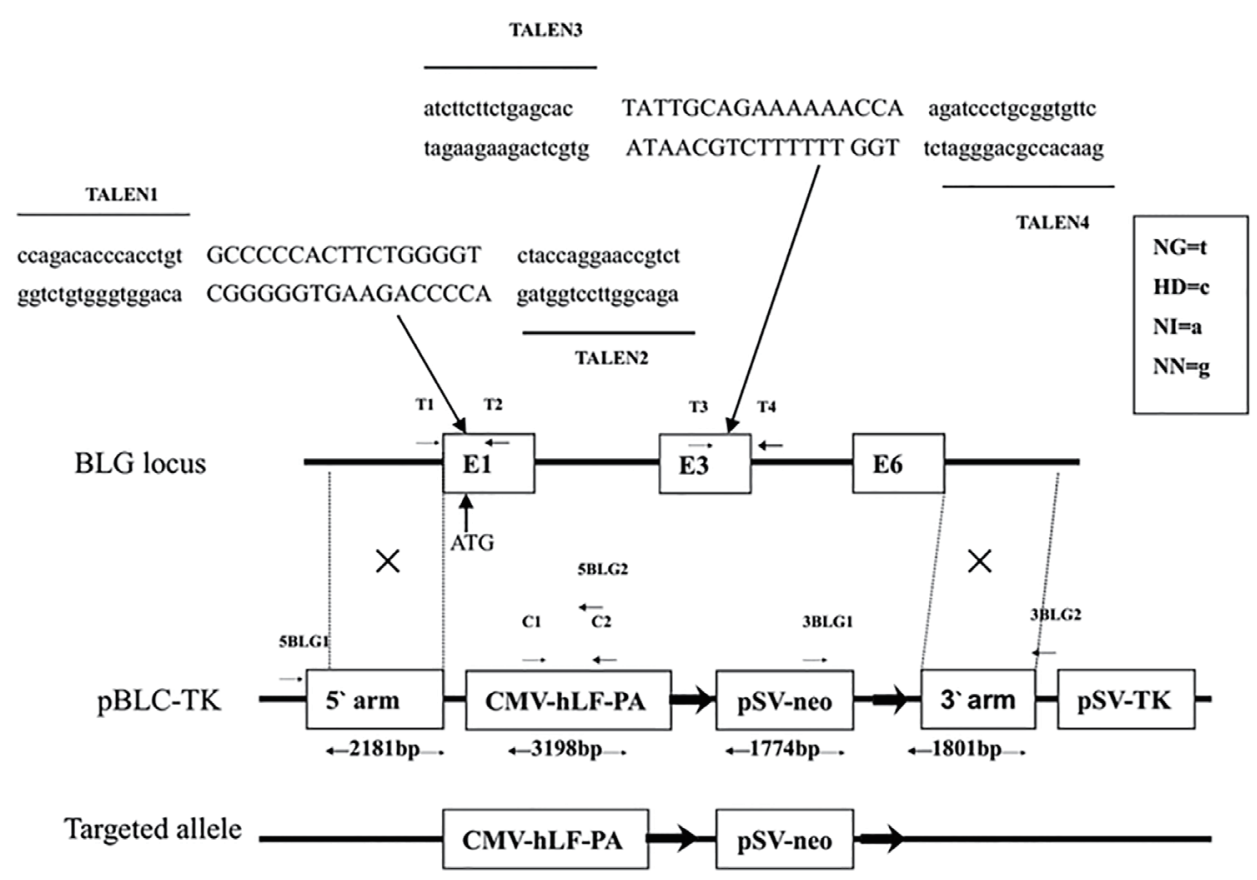

Figure 1. Schematic overview of the knock-in strategy for the beta-lactoglobulin (BLG) locus. Top, schematic representation of the BLG locus and the targeting vector with the human lacotoferrin (hLF) CDNA and bovine growth hormone polyadenylation signals. Bottom, schematic of the targeted integration of CMV-hLF gene and pSV-neo cassette. The black arrow represents Loxp locus.

products following the formula:

$$
\operatorname{Indels}(\%)=100 \times\left(1-\sqrt{1-\frac{b+c}{a+b+c}}\right)
$$

a: fraction of undigested PCR product; b, c: fraction of each cleavage product.

\section{Analysis of targeted clone and SCNT-derived fetus}

Linearized targeting construct (10 $\mu$ g, TakaRa, Shiga, Japan) and each TALEN expression plasmid $(15 \mu \mathrm{g})$ were electroporated to passage one cells $\left(5 \times 10^{6}\right)$, and then seeded in 6-well plates $(1.5 \times$ $10^{5}$ cells/well $)$ and puromycin selection $(1 \mu \mathrm{g} / \mathrm{mL})$ was applied at $24 \mathrm{~h}$ post-seeding. Later, from $48 \mathrm{~h}$ onward cells were cultured in selective medium containing $600 \mu \mathrm{g} / \mathrm{mL}$ G418 (TakaRa, Japan) and $2 \mu \mathrm{g} / \mathrm{mL}$ ganciclovir (TakaRa, Japan). At $10 \mathrm{~d}$ of culture, drugresistant colonies were ring-cloned for expansion and then used for downstream analysis. Accordingly, DNA was isolated in 96well plates or from the cell of SCNT-derived fetus by overnight lysis (50 mM Tris, pH 8, $20 \mathrm{mM}$ ethylenediamine tetraacetate, $100 \mathrm{mM} \mathrm{NaCl}, 0.3 \%$ sodium dodecyl sulfate, $10 \mathrm{mg} / \mathrm{mL}$ proteinase $\mathrm{K}$ ), then precipitated using isopropanol, and finally the pellets were resuspended in $50 \mu \mathrm{L}$ TE buffer $(10 \mathrm{mM}$ Tris- $\mathrm{HCl}$, $1 \mathrm{mM}$ ethylenediaminetetraacetic acid, $\mathrm{pH} 8$ ). To confirm the integration of transgene, first PCR was performed using primer sets C1/C2 (sense: 5'-ATAGTAACGCCAATAGGGA-3'; antisense: 5'-GGTCGCAGTTTGTAGGG-3'; 775-bp product) and second PCR was performed using primer sets 5BLG1/5BLG2 (sense: 5'-TGAAGTCATAAAGCCCGATACCTCC-3'; antisense:5'-ACAGCAGGA CGAGGAAGACAAGTTT-3'; 5' arm, 4,708-bp product) and 3BLG1/3BLG2 (sense: 5'- GCCGAGAA AGTATCCATCA-3'; antisense:5'- AGGGTGTTCCCAGGTCA -3'; 3' arm, 2,423-bp product) using DNA from PCR positive clone and SCNT-derived fetus. Purified PCR products were again cloned into pMD19-T (TakaRa, Japan) TA vectors (Sheng gong, ShangHai, China) and sequenced with primers 5BLG1 and 3BLG2 to further confirm gene targeted at the BLG locus.

Somatic cell nuclear transfer

The gene targeted fibroblasts were inserted into enucleated oocytes by SCNT. F1 fibroblasts were isolated from $43 \mathrm{~d}$ fetus of one pregnancy and conducted secondary SCNT using F1 fibroblasts as donors according to the protocol descrived above [17]. Consisely, oocytes enucleation was performed under UV light, and a single fibroblast cell was injected into the perivitelline space of an enucleated oocyte in $\mathrm{M}_{2}$ (Sigma, USA) medium supplemented with 10\% FCS (HyClone, Beijing, China), $2 \mu \mathrm{g} / \mathrm{mL}$ Hoechst 33342 (Sigma, USA) and $7.5 \mu \mathrm{g} / \mathrm{mL}$ cytochalasin B (Sigma, USA). Fusion of cell-oocyte couplets was accomplished by providing two direct current pulses $(1.5 \mathrm{kV} / \mathrm{cm}$ for $40 \mu \mathrm{s})$ using an electrical fusion solution containing $0.3 \mathrm{M}$ mannitol, $0.5 \mathrm{mM}$ HEPES, 0.05 $\mathrm{mM} \mathrm{CaCl}_{2}$, and $0.1 \mathrm{mM} \mathrm{MgSO}_{4}$ (Sigma, USA). After that, reconstructed oocytes were cultured in $\mathrm{M}_{16}$ medium containing $7.5 \mu \mathrm{g} / \mathrm{mL}$ cytochalasin B and $5 \mathrm{mM}$ ionomycin (Sigma, USA) for $5 \mathrm{~min}$, and then washed with fresh $\mathrm{M}_{16}$ medium and cultured for another $5 \mathrm{~h}$ in $\mathrm{M}_{16}$ medium containing $7.5 \mu \mathrm{g} / \mathrm{mL}$ cytochala- 
$\sin \mathrm{B}$ and $2 \mathrm{mMN}-6$ dimethylaminopurine (Sigma, USA). Finally, obtained reconstructed embryos were maintained in $\mathrm{M}_{16}$ medium for around $10 \mathrm{~h}$ untill they were transplanted to a naturally cycling surrogate recipients on the second day of estrus. Recipients were subjected to a transvaginal ultrasonographic evaluation at $30 \mathrm{~d}$ and $60 \mathrm{~d}$ of gestation.

\section{RESULTS}

\section{Construction of targeted vector}

A gene-targeting construct termed pBLC-TK (Figure 1) encompassing hLF cDNA was delivered to recombine at exon 1 and intron 5 of the caprine $B L G$ gene, resulting in the integration of a 5-kb fragment and subsequent disruption of endogenous caprine $B L G$ gene expression. Specially, hLF cDNA was directed to express in milk using a mammary specific caprine alpha-lactalbumin promoter/regulatory region which was reported as an efficient driver of transgene expression into milk of mice [18]. Neomycin resistance (neo) gene was used as a positive selection marker and a herpes simplex virus type 1 thymidine kinase (tk) gene was used as a negative selection marker in the construction of pBLC-TK knockout vector. The hLF cDNA and neo gene were flanked by two homologous arms, $3.7 \mathrm{~kb}$ for the $5^{\prime}$ arm and 1.8 $\mathrm{kb}$ for the $3^{\prime} \mathrm{arm}$. The loxP sites were arranged to flank the neo gene, which ultimately resulted in the removal of gene after Cremediated recombination.

Evaluation of non-homologous end joining-mediated gene disruption efficiency by TALEN

Two pairs of TALENs were designed to stimulate homologydirected repair (HDR), specifically to target the caprine BLG locus in the exon 1/3 (Figure 1). Caprine fetal fibroblasts were trans- fected with left and right TALEN plasmid to evaluate the targeting rate of the TALENs. Meanwhile, mutation was confirmed by PCR amplification of the desired site using DNA templates extracted from mixed TALENs transfected cells. Thereafter, a 729-bp DNA fragment (using prime T1/2 or T3/4) spanning the target site from mixed cells was cloned into pGEM-T Easy Vector (Promega, Madison, WI, USA) for sequencing. As shown in Figure 2A, targeting efficiency (TALEN1/2) has been confirmed of over $20 \%$ by the presence of double peaks after targeting site in the sequencing curves according to analysis of the company soft, a distinguishing feature of mutants from non-targeted cells. TALEN3/4 is proved to be less effective than TALEN1/2 (Figure $2 B)$. Sequencing of clones $(n=20)$ containing the amplified region showed a $13 \%$ deletion frequency at the target site (Figure $2 \mathrm{~A}, 2 \mathrm{~B})$. Beside this, some small deletions were detected in the TALEN 1/2 (left) and TALEN 3/4 (right) target regions (Figure 2C, 2D).

A nuclease assay was performed on mixed cells at $2 \mathrm{~d}$ of electroporation and was expected to generate two fragments of around $477 \mathrm{bp}$ and $252 \mathrm{bp}$ in clones with successful mutations at BLG target sequence. As expected, two separate bands of approximately $477 \mathrm{bp}$ and $252 \mathrm{bp}$ were detected in the TALENs-treated cell-derived amplification (Figure 3). The amplicon derived from wild-type cells possessed a single band at around $729 \mathrm{bp}$ (prime T1/2 and T3/4). Hence, the presence of mismatch DNA in the TALENs-treated cell-derived amplification further confirmed mutations induced by TALENs. Thus, use of TALENs bears enormous prospects for the induction of cleavage and mutation in target sequences. Moreover, the reveal of $10 \%$ T7EI activity in T7EI assay (Figure 3) indicated that $10 \%$ mutated alleles are in the cell pool and this can also prove that the TALEN pairs had a significant effect on nuclease activity in fibroblasts.
A

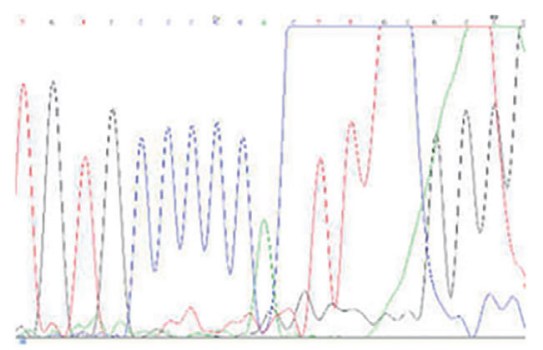

C
B

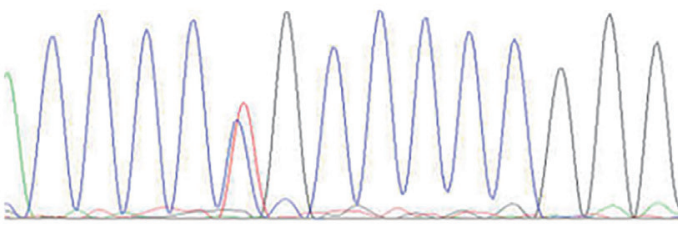

D

$\begin{array}{cc}\text { TATTGCAGAAAAAACCA } & \text { WT } \\ \text { TATTG - AGAAAAAACCA } & -1 \mathrm{bp} \\ \text { TATTGCAG - - - AACCA } & -4 \mathrm{bp}\end{array}$

Figure 2. Mutations induced by TALENs in goat fibroblasts. The graphs of sequencing modified alleles in fetus cell in which overlapped peaks were observed by using TALEN $1 / 2$ (A) and TALEN 3/4 (B). TALEN-induced mutations in BLG with 1/2 (C) and TALEN 3/4 (D). TALENs, transcription activator-like effector nucleases. 


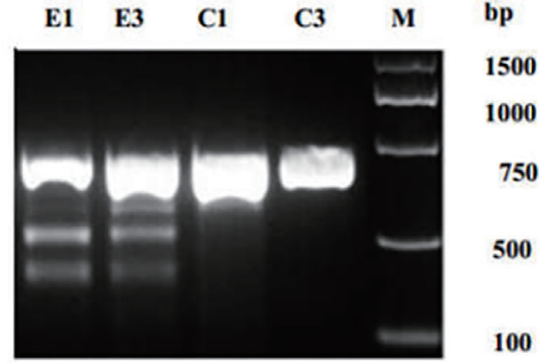

Figure 3. T7EI digestion of PCR products from the TALENs treated cells. E1: cells treated by TALENs of exon1; E3: cells treated by TALENs of exon3; C1, C3: untreated cells; M: marker. T7El, T7 endonuclease I; PCR, polymerase chain reaction; TALENs, transcription activator-like effector nucleases.

\section{Gene targeting of BLG locus in GFFs}

From above, cells were co-transfected with circular TALENs and linearized pBLC-TK, plated on 6-well plates and maintained at $37^{\circ} \mathrm{C}$, under puromycin selection. As TALENs plasmid containing puromycin gene express puromycin in the cytoplasm upon introduction of circular TALENs into the cytoplasm, the successfully transfected cells should not dye. After $48 \mathrm{~h}$ selection, about 8 to 10 cells in one 6-well plates grew as colonies and culture was continued with positive and negative selection untill the ringcloning was performed between 10 to 14 days of post-transfection to select individual clones. Each colony was expanded and individual clones were duplicated, one for cryopreservation and another for DNA extraction. One hundred and thirty-four drugresistance cell clones were obtained, where, $10^{4}$ cell clones were confirmed to harbor the $h L F$ gene by PCR using primer C1/C2 (Figure 4). Whereas, several cell clones went to apoptosis and could not be used for subsequent assays. Perfectly targeted clones were then identified by both PCR and sequencing analysis using 5BLG1/5BLG2 ( ${ }^{\prime}$ arm, 4,708-bp product) and 3BLG1/3BLG2 ( $3^{\prime}$ arm, 2,423-bp product) (Figure 5). Monoallelic and bi-allelic targets were also distinguished through analyzing the positive clones for the presence of the unmodified allele using primers (5BLG1/T2). PCR product of $4.5 \mathrm{~kb}$ was expected if $5.9 \mathrm{~kb}$ sequence was not removed. Consequently, bi-allelic recombination efficiency was $23 \%$ (8/35 clones) for the pBLC-TK and none of the positives clones had monoallelic modification.

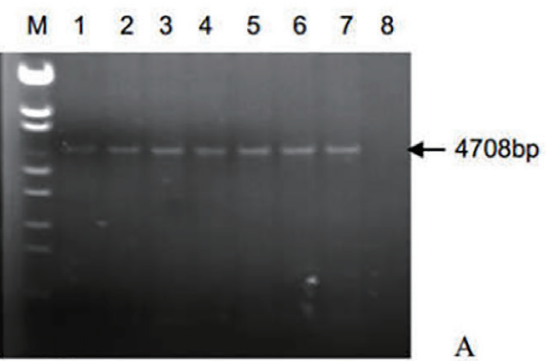

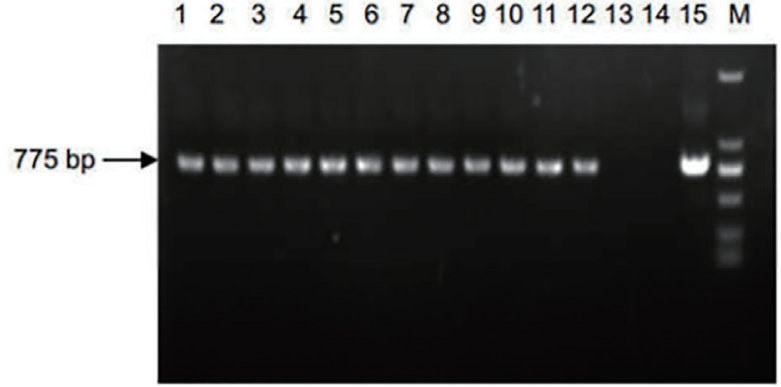

Figure 4. Polymerase chain reaction analysis of transgenic cell and fetus with primer C1 and C2. 1 to 11: targeted cell; 12: BK94 fetus; 13: negative cell; 14: Blank; 15: BLC-TK plasmid; M: marker 2000.

\section{Embryo transfer}

Five gene targeted clone cells were used in first round SCNT to produce transgenic goats. One hundred and eighty-two pronuclear-stage embryos were transferred into the oviducts of 17 recipient goats. Four $(23 \%)$ recipients were confirmed by ultrasound to maintain pregnancy at $30 \mathrm{~d}$ (Table 1). However, 3 out of 4 recipients aborted at 35 to $40 \mathrm{~d}$ and the remaining 45-day-old fetus (BK-94) was obtained surgically, which showed skin hyperaemia, and they were collected as donor cells for the second round SCNT. To confirm same genetic modification in the resulting fetus (BK-94) as it was found in the nuclear donors, PCR analysis was performed using the primer sets 5BLG1/5BLG2, 3BLG1/3BLG2, and C1/C2 to obtain a 4,708-bp, 2,423-bp, and 775-bp fragment corresponding to the $5^{\prime}$ promoter/regulatory region, $3^{\prime}$ untranslated region and $h L F$ gene, respectively (Figures 4-6). Also, the fetus was confirmed to be positive for the hLF target into BLG locus.

In second round SCNT, one hundred and fifty-five pronuclear-

Table 1. Efficiency of nuclear transfer from targeted cells

\begin{tabular}{lccccc}
\hline \multirow{2}{*}{ Cell clones } & $\begin{array}{c}\text { Number } \\
\text { of }\end{array}$ & $\begin{array}{c}\text { Number } \\
\text { of }\end{array}$ & & \multicolumn{3}{c}{ Number pregnant/total (\%) } \\
\cline { 5 - 6 } & embryos & recipients & Day 30 & Day 60 & Day 90 \\
\hline 12 & 182 & 17 & $4 / 17(23)$ & - & - \\
BK-94 & 155 & 13 & $7 / 13(53)$ & $4 / 13(31)$ & $3 / 13(23)$ \\
$\quad(45$ d targeted fetus) & & & & & \\
\hline
\end{tabular}

$\begin{array}{lllllllll}M & 1 & 2 & 3 & 4 & 5 & 6 & 7 & 8\end{array}$

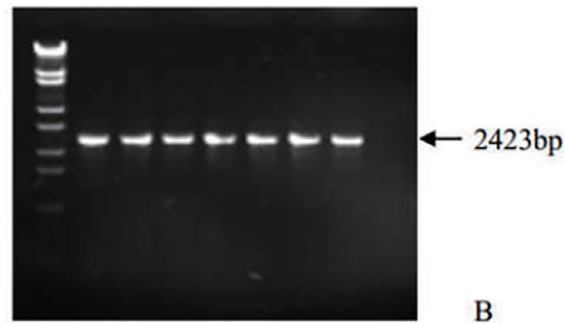

Figure 5. 5' and 3' Junction polymerase chain reaction analysis for screening the targeting events with primers. (A) 5BLG1 and 5BLG2; (B) 3BLG1 and 3BLG2. M: marker 2000; 1 to 6: a representative panel of 6 gene targeted cell clones; 7: BK94 fetus; 8: negative cell. 


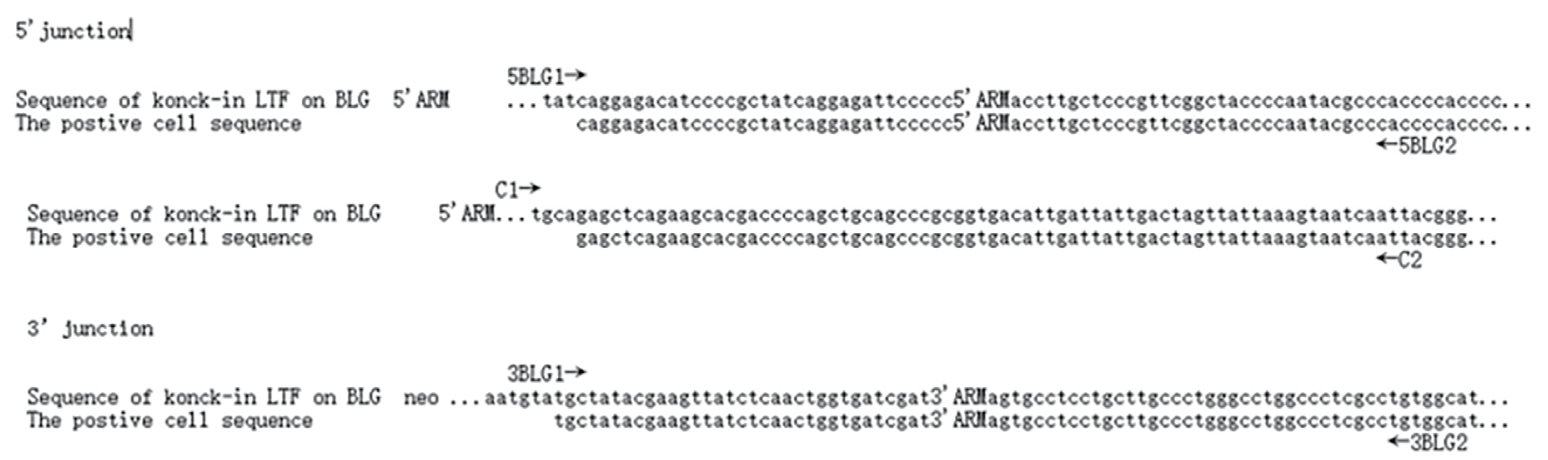

Figure 6. Sequence comparison at the $5^{\prime}$ and $3^{\prime}$ junctions with wild-type genomic DNA and donor DNA sequences of targeted goat and cell, respectively.

stage embryos were transferred into the oviducts of 13 recipient goats. Seven (53\%) and four (31\%) recipients were confirmed to be pregnant by ultrasound on $30 \mathrm{~d}$ and $60 \mathrm{~d}$, respectively. Interestingly, 3 recipients (48\%) maintained pregnant till $90 \mathrm{~d}$ and were confirmed by ultrasound. However, when the uteri was removed surgically, two recipients had uteri filled with $3 \mathrm{~kg}$-amniotic fluid and the other receipient had normal uterus at $150 \mathrm{~d}$ of gestation.

\section{DISCUSSION}

Zootechnical and reproductive characteristics of goats made it an attractive intermediate species between cows and rabbits with certain extent of superiority over sheep, such as better lactation performance. First, dairy goats produce more milk than rabbits and mice. Secondly, unlike cows, goats are known for having earlier sexual maturity, shorter breeding generation interval and more offspring per parity. Furthermore, many recombinant proteins such as antithrombin and albumin derived from transgenic goats are the first FDA approved and commercialized animal bioreactor or extraordinarily processed in clinical research [20]. Similarly, SCNT derived transgenic cows or goats expressing the recombinant human lactoferrin (rhLF) in the milk have also been characterized extensively $[15,16]$. However, previously reported transgene was simply the random integration of donor cell, where rhLF has been reported to express in goats with hLF mono-allelic targeted into BLG locus by two round step of gene targeting, and the expression level of rhLF in the milk was $3.2 \mathrm{mg} / \mathrm{mL}$. Our current transgenic strategy addresses this issue in a single step by replacing bi-allelic BLG with hLF in the BLG locus using TALENs, which can reduce the time and cost. Furthermore, the sequence between exon 1 and exon 6 was deleted in targeted cells.

As an alternative, application of ZFNs/TALENs/cas9 in cultured cells has also demonstrated a great promise for the creation of livestock with precise modifications. For example, high-efficiency disruption of BLG was achieved when ZFNs/TALENs/ cas9 plasmid or encoding mRNA were inserted into bovine or caprine fetal fibroblasts and embryos [12,21]. Although, successful mutation was not detected by TALENs, but, TALENs greatly stimulated HR after co-transfection with short or longer homol- ogous arm and application of positive and negative antibiotic selection strategy [9]. In current study, two pairs of TALEN expression plasmids were designed to target exon 1 and exon 3 of the BLG genes in goats. The short homologous arms (about $2 \mathrm{~kb}$ ) were used. First, the efficiency of TALEN-mediated gene mutation was detected and results found $20 \%$ targetting efficiency according to the sequencing map. Another important drawback of nucleases mediated genome editing is the off-target activity [22]. However, there was no identified off-target cleavage of the 2 pairs of TALENs used in our experiment. Certainly, lack of off-target cleavage is an outstanding advantage of TALENs over ZFNs and CRISPR/Cas9 system, while the later two techniques were reported to cause high off-target mutagenesis. Taking the data all together into consideration, it could be summarized that the TALENs is a reliable gene targeting tool for high efficient genetic modification in caprine somatic cells. Again, site-specific knock-in is another advantage of nuclease-mediated gene targeting in large animals. Co-transfection of TALENs with the linearized targeting construct directed HR for efficient insertion of hLF into BLG locus. Besides, both colonies were found to be bi-allelical modified, demonstrating the establishment of TALEN mediated gene targeting in our lab.

Exogenous DNA could be inserted into a specific site of the genome by TALEN-mediated gene targeting, and efficient selection of gene targeted clone cells could be performed by dual selectable marker genes. However, the integration of selectable marker genes may cause several problems, including public concerns regarding the release of antibiotic-resistance genes into environment and confounding the food safety evaluation of resultant transgenic animals [9]. In present study, considering the health concerns of both transgenic animals and humans, we introduced the loxP sites to flank the selectable marker genes, which will allow the removal of antibiotic-resistant marker genes by cre-mediated recombination in the future study [23].

In reproductive cloning by SCNT, the epigenetic signature of a differentiated somatic cell must be reset in a state resembling to totipotency and capability of driving full development after fusion of the cell to an enucleated oocyte cytoplast. Incomplete nuclear reprogramming is one of the major contributors to the 
low developmental success rate following SCNT [24]. Our results found $23 \%$ pregnancy at $30 \mathrm{~d}$ when the gene-targeted cell was used as donor cell in the first round SCNT. While, using gene targeted fetus cell at second round SCNT, the pregnancy rate at $30 \mathrm{~d}$ was significantly improved compared to the first round SCNT $(\mathrm{p}<0.01)$, which clearly indicating the recovering of genetic programming process at least in certain level. However, abortion of 13 recipients in between $60 \mathrm{~d}$ and $90 \mathrm{~d}$ pregnancy revealing that the gene targeting process is more complex than general cloning techniques. Gene targeting not only suffer reprogramming in SCNT, but also endure intense pressure from HR and cell selection. Abnormal epigenetic modifications such as DNA methylation and histone modifications might serve as a key factor affecting cloning efficiency in gene targeted or cloned embryos and fetuses [25-27]. Potentially, the problem might be the result of bi-allelic gene targeted into BLG locus. Hence, further study should be done to increase the developmental capacity of cloned embryos by enhancement of nuclear reprogram ability of donor cells and embryos in combination with other factors such as histone deacetylase inhibitor [25] and removal of $\mathrm{H} 3 \mathrm{~K} 9 \mathrm{me} 3$ in donor somatic cells by ectopically expressed H3K9me3 demethylase such as Kdmd [28].

In conclusions, we have demonstrated that constructed TALENs can be used to stimulate HDR significantly in fetus fibroblast cell, leading to the bio-allelic introduction of hLF into BLG locus in one step. Also, the establishment of the bio-allelic gene targeted fetus fibroblast cell can be used for further genetic engineering to produce transgenic goats. In addition, we have shown that such gene targeted cells are capable of producing pregnancy (recloning) for at least $90 \mathrm{~d}$.

\section{CONFLICT OF INTEREST}

We certify that there is no conflict of interest with any financial organization regarding the material discussed in the manuscript.

\section{ACKNOWLEDGMENTS}

This study was supported by A Project Funded by the Priority Academic Program Development of Jiangsu Higher Education Institutions (PAPD), China Postdoctoral Science Foundation (2015M571827/1402001C), Inter governmental science and technology cooperation project (S2016G6252) and Jiangsu Province Science and Technology Support Project (BE2013679).

\section{REFERENCES}

1.Meyer M, de Angelis MH, Wurst W, Küh R. Gene targeting by homologous recombination in mouse zygotes mediated by zinc-finger nucleases. Proc Natl Acad Sci USA 2010;107:15022-6.

2.McCreath KJ, Howcroft J, Campbell KH, et al. Production of genetargeted sheep by nuclear transfer from cultured somatic cells. Nature
2000;405:1066-9.

3.Liu X, Wang Y, Guo W, et al. Zinc-finger nickase-mediated insertion of the lysostaphin gene into the beta-casein locus in cloned cows. Nat Commun 2013;4:2565.

4.Liu X, Wang Y, Tian Y, et al. Generation of mastitis resistance in cows by targeting human lysozyme gene to $\beta$-casein locus using zinc-finger nucleases. Proc Biol Sci B 2014;281:20133368.

5.Zhang X, Wang L, Wu Y, et al. Knockout of myostatin by zinc-finger nuclease in sheep fibroblasts and embryos. Asian-Australas J Anim Sci 2016;29:1500-7.

6.Yu B, Lu R, Yuan Y, et al. Efficient TALEN-mediated myostatin gene editing in goats. BMC Dev Biol 2016;16:26.

7.Proudfoot C, Carlson DF, Huddart R, et al. Genome edited sheep and cattle. Transgenic Res 2015;24:147-53.

8.Carlson DF, Tan W, Lillico SG, et al. Efficient TALEN-mediated gene knockout in livestock. Proc Natl Acad Sci USA 2012;109:17382-7.

9.Cui C, Song Y, Liu J, et al. Gene targeting by TALEN-induced homologous recombination in goats directs production of $\beta$-lactoglobulinfree, high-human lactoferrin milk. Sci Rep 2015;5:10482.

10. Sharma S, Pravindra K, Betzel C, Singh TP. Structure and function of proteins involved in milk allergies. J Chromatogr B Biomed Sci Appl 2001;756:183-7.

11. Yu, S, Luo J, Song Z, et al. Highly efficient modification of beta-lactoglobulin $(B L G)$ gene via zinc-finger nucleases in cattle. Cell Res 2011; 21:1638-40.

12. Jabed A, Wagner S, McCracken J, Wells DN, Laible G. Targeted microRNA expression in dairy cattle directs production of $\beta$-lactoglobulinfree, high-casein milk. Proc Natl Acad Sci USA 2012;109:16811-6.

13. Yang B, Wang J, Tang B, et al. Characterization of bioactive recombinant human lysozyme expressed in milk of cloned transgenic cattle. PLoS One 2011;6:e17593.

14. Yu H, Chen J, Sun W, et al. The dominant expression of functional human lactoferrin in transgenic cloned goats using a hybrid lactoferrin expression construct. J Biotechnol 2012;161:198-205.

15. Yang P, Wang J, Gong G, et al. Cattle mammary bioreactor generated by a novel procedure of transgenic cloning for large-scale production of functional human lactoferrin. PLoS One 2008;3:e3453.

16. An LY, Yuan YG, Yu BL, Yang TJ, Cheng Y. Generation of human lactoferrin transgenic cloned goats using donor cells with dual markers and a modified selection procedure. Theriogenology 2012;78:1303-11.

17. Cheng Y, An LY, Yuan GY, et al. Hybrid expression cassettes consisting of a milk protein promoter and a cytomegalovirus enhancer significantly increase mammary-specific expression of human lactoferrin in transgenic mice. Mol Reprod Dev 2012;79:573-85.

18. Bertolini LR, Meade H, Lazzarotto CR, et al. The transgenic animal platform for biopharmaceutical production. Transgenic Res 2016;32: 107-21.

19. Guschin DY, Waite AJ, Katibah GE, et al. A rapid and general assay for monitoring endogenous gene modification. Methods Mol Biol 2010;649:247-56.

20. Xu Y, Liu S, Yu G, et al. Excision of selectable genes from transgenic goat cells by a protein transducible TAT-Cre recombinase. Gene 2008; 
419:70-4.

21. Yu H, Chen J, Liu S, et al. Large-scale production of functional human lysozyme in transgenic cloned goats. J Biotechnol 2013;168:676-83.

22. Cho SW, Kim S, Kim Y, et al. Analysis of off-target effects of CRISPR/ Cas-derived RNA-guided endonucleases and nickases. Genome Res 2014;24:132-41.

23. Lina L, Xu W, Dai Y, Li N. DNA methylation changes in cell line from $\beta$-lactoglobulin gene targeted fetus. Anim Reprod Sci 2009;112:402-8.

24. Bui HT, Wakayama S, Kishigami S, et al. Effect of trichostatin A on chromatin remodeling, histone modifications, DNA replication, and transcriptional activity in cloned mouse embryos. Bio Reprod 2010;83: 454-63.
25. Huan Y, Zhu J, Huang B, et al. Trichostatin A rescues the disrupted imprinting induced by somatic cell nuclear transfer in pigs. PLoS One 2015;10:e126607.

26. Meng L, Wan Y, Sun Y, et al. Generation of five human lactoferrin transgenic cloned goats using fibroblast cells and their methylation status of putative differential methylation regions of IGF2R and H19 imprinted genes. PLoS One 2013;8:e77798.

27. Tian XC. Genomic imprinting in farm animals. Annu Rev Anim Biosci 2014;2:23-40.

28. Matoba S, Liu Y, Lu F, et al. Embryonic development following somatic cell nuclear transfer impeded by persisting histone methylation. Cell 2014;159:884-95. 